\title{
Developing a novel inhaled therapeutic for the treatment of bronchiolitis obliterans syndrome
}

Breath Therapeutics - a Zambon Group Company, is a clinical stage biopharmaceutical company specialising in advanced hiled therapeutics The company's propriotary liposomal drug formulation has been specifically designed for inhaled administration with an exclusively licensed nebulizer technology. Liposomal cyclosporine $A$ for inhalation (L-CsA-i), an investigational drug, is advancing in phase 3 clinical trials as the first poten
therapy for bronchiolitis

and devastating lung diseas with no approved treatments.

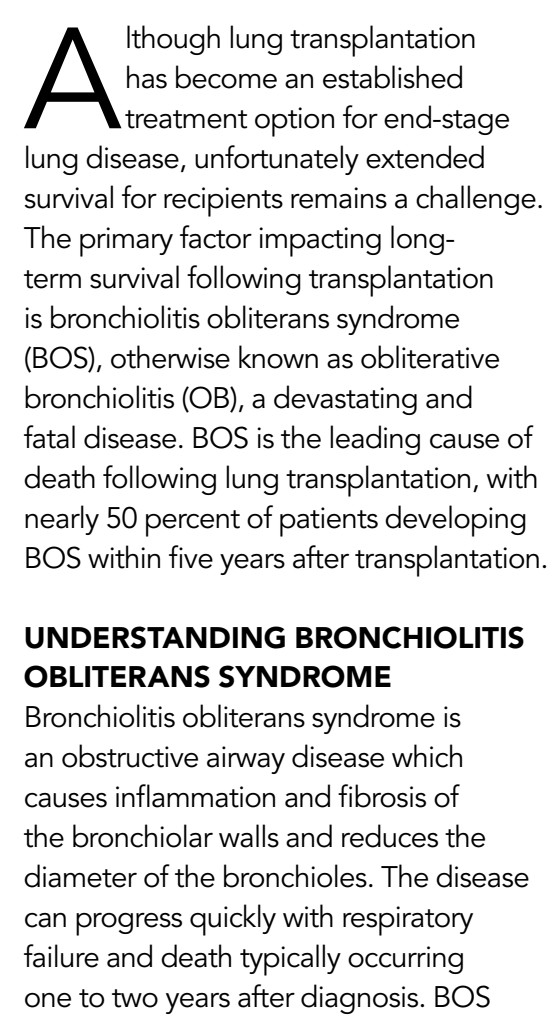

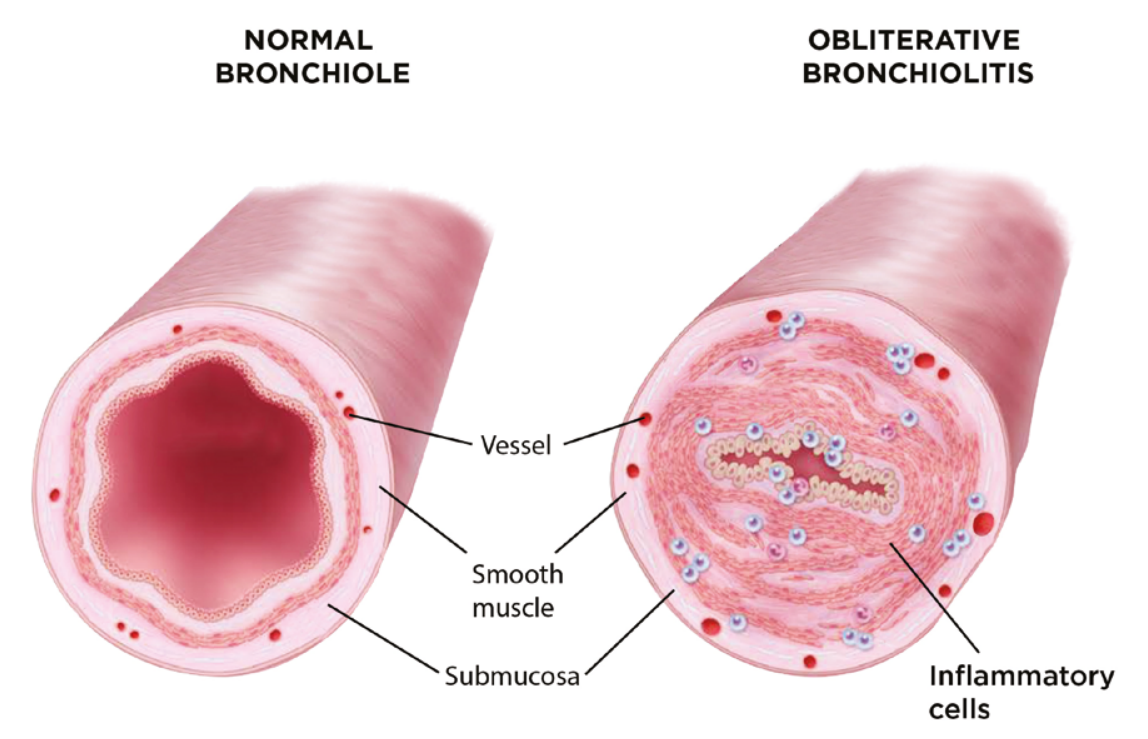

is commonly seen in patients following lung transplantation and allogeneic hematopoietic stem cell transplant (alloHSCT), although it is also associated with autoimmune disease and exposure to environmental contaminants. Based on 21,500 mant research, an estimated alloHSCT recipients are currently affected by BOS in the US, Europe and afen. Alongside the physical consequences for

\section{BOS can progress} quickly with respiratory failure and death typically occurring one to two years after diagnosis.

patients with BOS and potential negative impact on the mental well-being of both there is also an economic burden of the disease. US helthcre utilisation data shows a 30-40 percent annual increase in costs in treating lung transplant recipients with BOS compared to lung transplant patients without the disease. Despite this significant humanistic and economic burden, there are no approved treatments for BOS at this time.

ADVANCING INHALED RESPIRATORY THERAPY Breath Therapeutics, founded in 2016 and acquired by Zambon S.p.A. in July 2019, is led by Chief Executive Brer, Jens Stegemann, $\mathrm{FnD}$ and the Breath team consists of multi-skilled professionals with expertise in drug
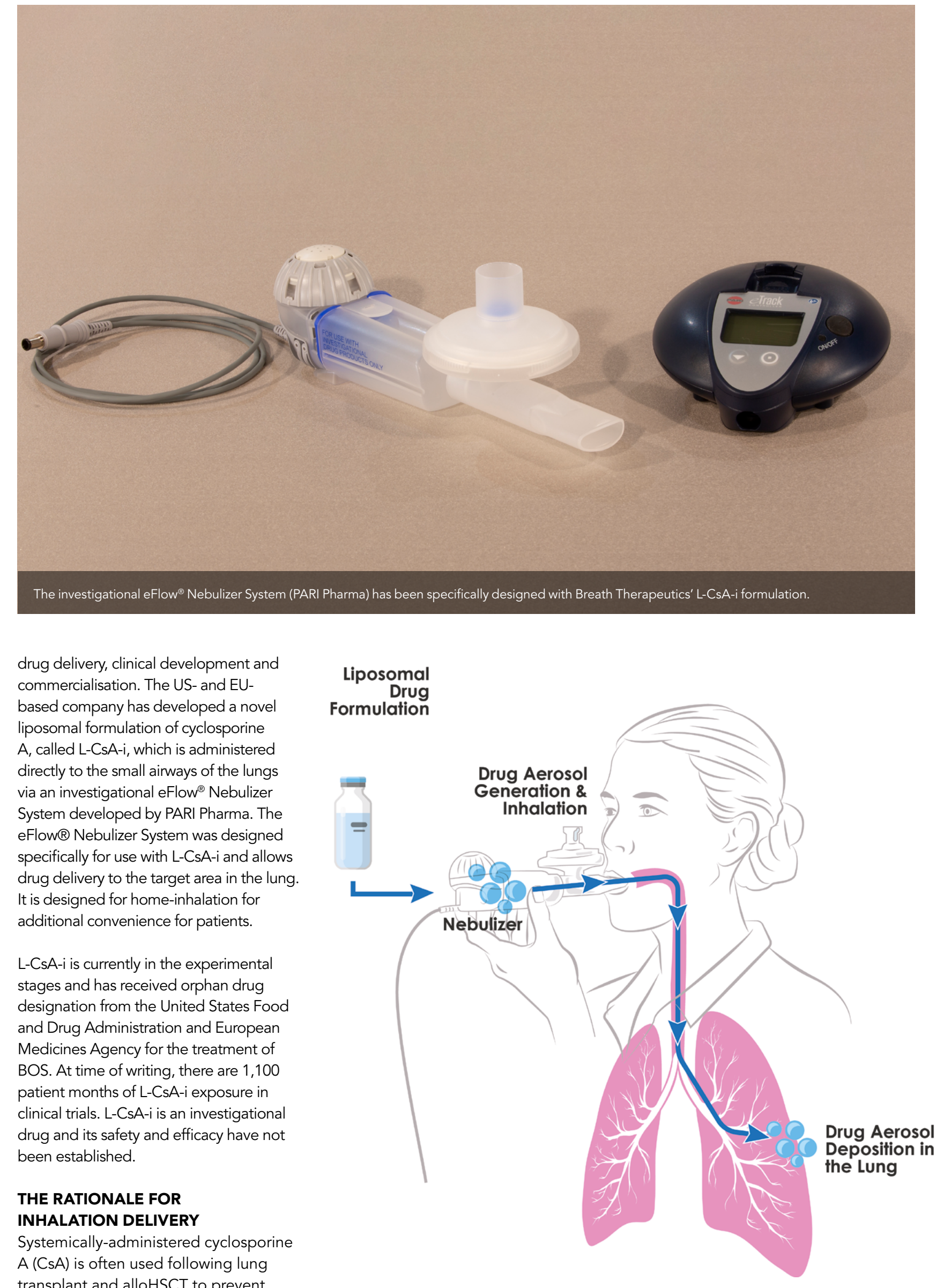
BOSTON DEVELOPMENT PROGRAM $\mathrm{L}-\mathrm{CSA}-\mathrm{i}^{*}$ for the Treatment of Bronchiolitis Obliterans Syndrome (BOS)

BOSTON-1

L-CsA-i for BOS following single lung transplant [Ph3] (Initiated Q1 2019)

\section{BOSTON-2}

L-CsA-i for BOS following double lung transplant [Ph3] (Initiated Q1 2019)

\section{BOSTON-3}

Extension trial of BOSTON-1 and BOSTON-2

\section{BOSTON-4}

L-CsA-i for BOS following alloHSCT*

\section{BOSTON-5}

L-CsA-i for pediatric patients with BOS

*Liposomal Cyclosporine A for Inhalation (L-CsA-i)

**Allogeneic Hematopoietic Stem Cell Transplantation (alloHSCT)

acute lung allograft rejection or acute and chronic graft versus host disease. In the systemic mode of administration at CA is not able to achieve sufficient Crug levels at the site of disease in the bronchioles for the treatment of BOS. The potential advantage of inhaled therapies is to deliver a drug directly to the site of the disease and to lower

L-CSA-i CLINICAL TRIALS FOR THE TREATMENT OF BOS The company is in the process of the safety and efficacy of $L-C s A-$.

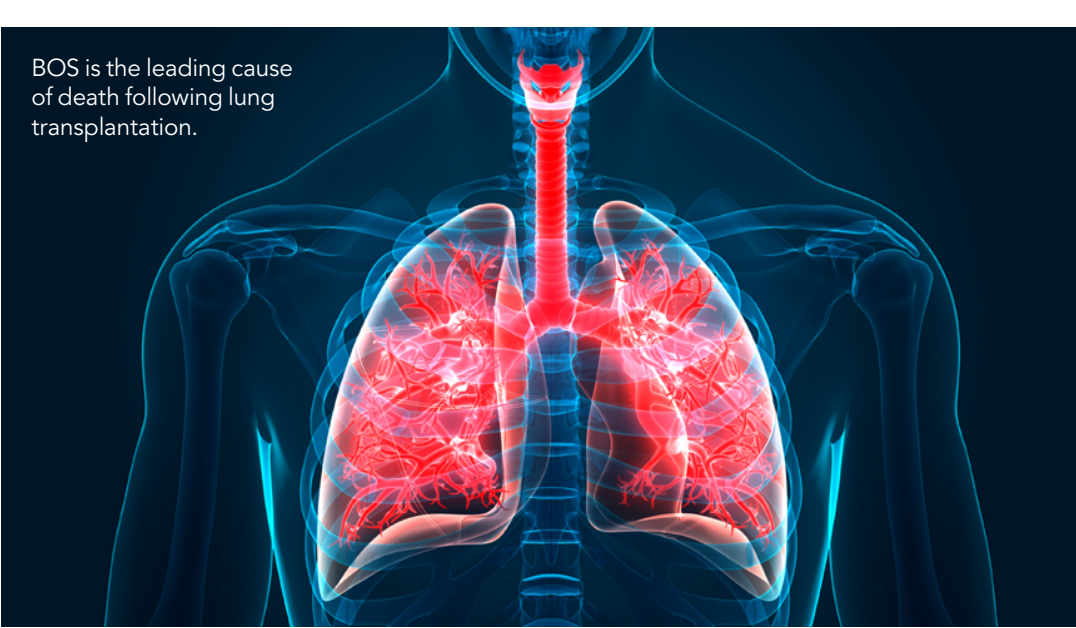

The BOSTON development program The BOSTON-1 and BOSTON-2 clinical aims to evaluate the use of $L-C S A-i \quad$ trials were initiated in March 2019.

The mission of Breath Therapeutics - a Zambon Group Company is to bring the first safe and effective treatment to people with BOS.

for the treatment of BOS in patients BOSTON-1 is evaluating L-CSA-i for the aged six and older. The BOSTON treatment of BOS program build upon prosTON that suggests local administration of immunosuppressives in the airways may be a potential option

in single lung transplant recipients whereas BOSTON-2 applies the same methodolog to double lung transplant recipients with participants at 35 leading lung transplant specialty centers in eight countries. Upon completion of the 48-week trials participants will be eligible to continue in BOSTON-3, an open-label extension trial of BOSTON-1 and BOSTON-2.

Two additional clinical trials are planned. The target participants for BOSTON-4 are adult recipients of alloHSCT. BOSTON-5 will study L-CsA-i in paediatric patients ages 6 to 17 years.

Breath Therapeutics - a Zambon Group Company is dedicated to developing a

\section{Behind the Research}

\section{Breath Therapeutics - a Zambon Group Company}

E: contact@breath-therapeutics.com W: www.breath-therapeutics.com

\section{Research Objectives}

Breath Therapeutics - a Zambon Group Company is developing therapies that combine novel formulations of existing drugs with inhalation technology for the treatment of rare respiratory diseases with high unmet medical need.

\section{Detail}

\section{Biography}

MD, Chief Medical Officer, Breath

The rapeutics - a Zambon Group Company. Prior, Dr Henis was Senior Director, Global Medical Affairs, Respiratory and PAH of Gilead Sciences. Previously, she held academic positions in respiratory diseases and lung transplant at California Pacific Medical Center and Stanford University School of Medicine. She earned her M.D. with Distinction in Immunology from Albert Einstein College of Medicine.

Dominik Kappeler, MD, Director Clinical Science \& Medical Monitor, Breath Therapeutics - a Zambon Group Company. Prior, he was head of the Clinical Unit at Inamed $\mathrm{GmbH}$, a respiratory focused CRO, and served as Principal Investigator in over 75 clinical trials. Previously, Dr Kappel worked for Servier and Harison Clinical Research (now Synteract). He graduated from Medical School at the School at the

Breath Therapeutics - a Zambon Group Company Adringenstraße 4

D0639 Munich, Germany

Breath Therapeutics - a Zambon Group Company 633 Menlo Avenue \#230

$\mathrm{T}:+16502720655$

\section{Collaborators}

Currently two Phase 3 studies are being conducted at up to 35 leading lung transplant centers in eight countries:

BOSTON-1 clinical study: https://clinicaltrials.gov/ct2/show/ NCT03657342? term =NCT03657342\&rank=1 /ct2/show/

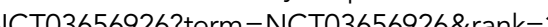

\section{References}

Meyer KC, et al. (2014). An international ISHLT/ATS/ERS clinical practice guideline: diagnosis and management of bronchiolitis obliterans syndrome. Eur Respir
1503. DOI: 10.1183/09031936.00107514.

Chambers DC, et al. (2018). The International Thoracic Organ Transplant Registry of the International Society for Deart and Lung Transplantation: Thirty-fifth adult lung and heart-lung transplant report-2018; Focus theme: Multiorgan doi: 10.1016/jihealun.2018.07.022.

International Society for Heart and Lung Transplantation 36(10):1037-1079. doi:10.1016/j.healun.2017.07.019.

Barker AF, et al. (2014). Obliterative Bronchiolitis. NEJM. 370:1820-8. doi:10.1056/NEJMra1204664.

Verleden GM, et al. (2019). Chronic lung allograft dysfunction: Definition, diagnostic criteria, and approaches to treatment - A consensus report from the Pulmonary Council of the SHLT. J Heart Lung Transplant. Vol 38, Issue 5, 493 - 503

\section{Personal Response}

What are your short-term and long-term goals for this drug candidate?

II Our immediate goal is completing the BOSTON-1 and for the treatment of BOS following lung transplantation. In parallel, we are preparing to initiate additional studies under our BOSTON development program.

At the same time, the commercial team is preparing to bring L-CSA-i to people with BOS upon receiving the geographies. Our ultimate mission is to offer the first safe and effective treatment to people with BOS, an underserved
community with an urgent unmet medical need.

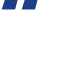

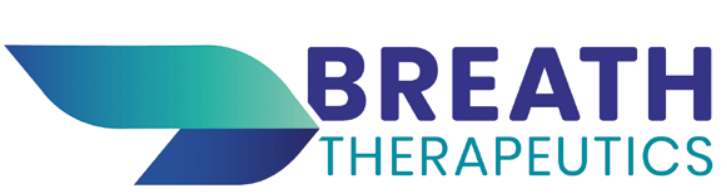

